\title{
Impact of Center Donor Acceptance Patterns on Utilization of Extended-Criteria Donors and Outcomes
}

\author{
Nicholas Hess ${ }^{1}$, Laura Seese ${ }^{2}$, Ibrahim Sultan ${ }^{3}$, Yisi Wang ${ }^{2}$, Floyd Thoma ${ }^{2}$, and Arman \\ Kilic $^{2}$ \\ ${ }^{1}$ University of Pittsburgh Medical Center \\ ${ }^{2}$ University of Pittsburgh Medical Center Health System \\ ${ }^{3}$ University of Pittsburgh
}

April 12, 2021

\begin{abstract}
Background: This study investigated the impact of transplanting center donor acceptance patterns on usage of extendedcriteria donors (ECDs) and posttransplant outcomes following orthotopic heart transplantation (OHT). Methods: The Scientific Registry of Transplant Recipients was queried to identify heart donor offers and adult, isolated OHT recipients in the United States from 1/1/2013-10/17/2018. Centers were stratified into 3 equal-size terciles based on donor heart acceptance rates $(<13.7 \%, 13.7 \%-20.2 \%,>20.2 \%)$. Overall survival was compared between recipients of ECDs ([?]40 years, left ventricular ejection fraction $<60 \%$, distance [?]500 miles, hepatitis B, hepatitis C or human immunodeficiency virus, or [?]50 offers) and recipients of traditional-criteria donors, and among transplanting terciles. Results: A total of 85,505 donor heart offers were made to 133 centers with $15,264(17.9 \%)$ accepted for OHT. High-acceptance programs $(>20.2 \%)$ more frequently accepted donors with LVEF $<60 \%$, HIV, HCV, and/or HBV, [?]50 offers, or distance >500 miles from the transplanting center (each $\mathrm{p}<0.001)$. Posttransplant survival was comparable across all three terciles $(\mathrm{p}=0.11)$. One- and five-year survival were also similar across terciles when examining recipients of all five ECD factors. Acceptance tier and increasing acceptance rate were not found to have any impact on mortality in multivariable modeling. Of ECD factors, only age [?]40 years was found to have increased hazards for mortality (HR 1.33, 95\% CI 1.22-1.46, p<0.001). Conclusions: Of recipients of ECD hearts, outcomes are similar across center-acceptance terciles. Educating less aggressive programs to increase donor acceptance and ECD utilization may yield higher national rates of OHT without major impact on outcomes.
\end{abstract}

\section{Introduction:}

Cardiac transplantation remains the gold standard treatment for end stage heart failure ${ }^{1}$, and despite the growing prevalence of advanced heart failure in the United States, donor heart supply critically limits rates of $\mathrm{OHT}^{2}$. This supply and demand mismatch is magnified by the fact that over $50 \%$ of offered donor hearts are not accepted for transplantation ${ }^{3}$. The reduced utilization of certain donor hearts is largely tied to the practice of rejecting organs that fall outside of strict donor selection criteria. This approach has been adopted from literature that suggests worse outcomes among recipients of certain donor types and concerns regarding regulatory reprimand for transplant centers with more pronounced mortality rates ${ }^{4}$. However, donor selection is more nuanced than the strict donor guidelines suggest.

In addition to published donor selection criteria, other features must be considered when selecting an appropriate donor. These include the clinical status and interactions of the donor-recipient pair, as well as the clinical volume and available infrastructure of the transplanting center. Although each of these components may impact the overall success of OHT with extended-criteria donor (ECD) hearts, higher transplant center volumes have been linked to improved survival over lower volume centers despite transplanting higher-risk 
recipients ${ }^{5-8}$. Furthermore, higher volume OHT centers may obtain a degree of their transplant volume by being more aggressive on the acceptance of donor hearts outside of the standard criteria. Considering the shortage of available donor hearts and that ECD hearts may be an option to expand the available donor pool, we investigated if the acceptance rates of ECD hearts varied among centers based on OHT volume and if the acceptance of these organs impacted outcomes.

\section{Materials and Methods:}

\section{Study cohort}

The Scientific Registry of Transplant Recipients (SRTR) was utilized to obtain data on all adult patients ([?]18 years) undergoing OHT in the United States between January 1, 2013 and October 17, 2018. Recipients were followed until April 1, 2020. Patients undergoing concomitant OHT with another organ (e.g. heartliver, heart-lung, heart-kidney etc.) were excluded. Additionally, we excluded all recipients who underwent OHT at centers performing less than 10 total OHTs during the study period. The following characteristics were used to identify ECDs: donor age [?]40 years, left ventricular ejection fraction (LVEF) $<60 \%$, distance from transplant center [?] 500 miles, hepatitis B (HBV), hepatitis C (HCV) or human immunodeficiency virus (HIV) positive, and donors with [?]50 offers prior to acceptance.

\section{Center-level data}

As mentioned, all patients undergoing OHT at centers performing fewer than 10 OHTs during the study period were excluded, and the corresponding centers were also excluded from the analysis. Transplant centers were stratified into three equal terciles based on OHT donor acceptance rates. Donor acceptance rates were calculated by taking the number of donor hearts accepted and dividing by the total number of offers. Donors were included in the analysis only if at least one solid organ was used for transplantation.

\section{Statistical analysis}

Continuous data are presented as mean \pm standard deviation for parametric data and median (interquartile range, IQR) for non-parametric data. Categorical data are presented as number (percentage). Student's t test, analysis of variance (ANOVA), and Mann-Whitney $U$ test were utilized for pairwise comparison of parametric and non-parametric data, as indicated. Risk adjusted post-OHT mortality was evaluated using multivariable Cox Frailty regression with random effects and transplant center as the nested variable. Statistical analyses were performed using the SAS version 9.4 software package (SAS Institute Inc, Cary, NC).

\section{Results}

Study population and Donor Acceptance Rates

A total of 85,505 donor heart offers were made to 133 different centers, which resulted in 15,264 (17.9\%) accepted donor hearts and 15,222 (17.8\%) OHTs during the study period. The average acceptance rate per center was $19.1 \pm 10.8 \%$ (Table 1 ). The median donor acceptance rate was $16.9 \%$ [interquartile range (IQR) 12.1\%-23.1\%]. The most common reasons for donor refusal were donor age in $53.9 \%(\mathrm{n}=32,731)$ and distance from transplanting center in $27.9 \%(\mathrm{n}=16,911)$.

Of accepted donor hearts, $9.3 \%(\mathrm{n}=3,697)$ over were from donors older than 40 years, $15.4 \%(\mathrm{n}=4,445)$ with LVEF $<60 \%, 3.9 \%(\mathrm{n}=737)$ over 500 miles from the transplanting center, and $14.7 \%(\mathrm{n}=604)$ with HIV, HCV or HBV. Lastly, $1.6 \%(\mathrm{n}=498)$ of donors with over 50 offers were ultimately accepted for OHT (Table 2 ).

\section{Donor Acceptance Stratified by Transplant Center Volume}

Transplant centers were stratified into 3 terciles by the center's donor acceptance rate, which included low-tier $(<13.7 \%)$, mid-tier (13.7\%-20.2\%), and high-tier (>20.2\%) (Table 3 ). Among the center terciles, mid-tier and high-tier programs were more likely to accept ECD organs. Specifically, donors over 40-years were most commonly accepted by mid-tier programs $(41.7 \%$ vs $48.2 \%$ vs $43.8 \%, \mathrm{p}<0.001)$. However, high-tier programs more frequently accepted donor offers with LVEF $<60 \%$ ( $8.9 \%$ vs $14.4 \%$ vs $26.2 \%, \mathrm{p}<0.001$ ), HIV, HCV, 
and/or HBV (6.9\% vs $11.4 \%$ vs $25.2 \%, \mathrm{p}<0.001)$, donors with [?]50 offers ( $0.6 \%$ vs $1.4 \%$ vs $3.4 \%, \mathrm{p}<0.001)$, and donors $>500$ miles from the accepting center $(1.3 \%$ vs $2.6 \%$ vs $8.7 \%, \mathrm{p}<0.001)$. Despite higher rates of acceptance in mid- and high-tier programs, in some categories, such as patients with LVEF $<60 \%$, low-tier programs had a higher volume of donor offers.

\section{OHT Outcomes Stratified by Donor Acceptance Tercile}

The rates of 30 -day ( $3.6 \%$ vs $3.9 \%$ vs $3.4 \%, \mathrm{p}=0.44)$ and 1 -year $(9.5 \%$ vs $8.7 \%$ vs $8.7 \%, \mathrm{p}=0.29)$ mortality were comparable among the three terciles (Table 4 ). The rates of post-OHT stroke, retransplantation, permanent pacemaker placement, and cardiac reoperations were similar between the terciles. The post-transplant length of stay $(24.0[+-29.2]$ vs 22.3 [+- 24.3] vs 21.1 [+- 22.9] days, $\mathrm{p}<0.001)$ was longest in the lowest tercile. Rates of renal failure requiring dialysis $(12.57 \%$ vs $13.99 \%$ vs $12.38 \%, \mathrm{p}=0.03)$ and drug treated rejection $(12.19 \%$. vs $12.83 \%$ vs $9.89 \%, \mathrm{p}<0.001)$ were highest in the middle tercile. The tercile-specific survival and retransplantation rates were similar among recipients who received donor hearts with LVEF $<60 \%$, HIV, HCV or HBV, $>500$ miles from the transplant center, and those with $>50$ offers (Supplemental Table 1 ). However, recipients of older donor hearts ( $>40$ years) had similar survival between the terciles but higher rates of retransplantation in the highest tercile.

\section{Associations between Mortality and Extended Risk Criteria}

We compared the outcomes of the recipients receiving ECD hearts to those who received traditional-criteria donor hearts. We noted similar long-term mortality between the recipients of donor hearts with LVEF $<60 \%$ compared to those with LVEF $>60 \%$ ( $15.3 \%$ vs $14.8 \%, \mathrm{p}=0.52)$, those with $>50$ offers compared to [?] 50 offers $(15.1 \%$ vs $16.7 \%, \mathrm{p}=0.32)$, HCV, HBV, and HIV positive versus negative donors $(15.2 \%$ vs $13.2 \%$, $\mathrm{p}=0.18)$, and donors $>500$ compared to [?]500 miles from the transplant center $(15.1 \%$ vs $16.0 \%, \mathrm{p}=0.51)$ (Supplemental Table 2 ). However, when compared to recipients of donors [?]40 years, recipients of donor hearts $>40$ years of age had higher rates of overall post-OHT mortality $(14.1 \%$ vs $18.4 \%, \mathrm{p}<0.001)$.

\section{Recipient Characteristics Stratified by Center Tercile}

Recipients in low-tier centers were younger at listing (55 years [IQR 44-62] vs 56 years [IQR 46-63] vs 56 years [IQR 47-63], $\mathrm{p}<0.001$ ) (Supplemental Table 3). Furthermore, low-tier recipients were more likely to be black race $(26.6 \%$ vs $21.9 \%$ vs $20.8 \%, \mathrm{p}<0.001)$, to have less education than a college degree $(52.3 \%$ vs $54.5 \%$ vs $60.9 \%, \mathrm{p}<0.001)$, to be bridged to transplant with an LVAD $(54.1 \%$ vs $52.0 \%$ vs $35.7 \%, \mathrm{p}<0.001)$, and to have the lowest serum bilirubin levels $(0.60 \mathrm{mg} / \mathrm{dL}$ [IQR $0.4-1.0]$ vs $0.7 \mathrm{mg} / \mathrm{dL}$ [IQR $0.4-1.0$ ] vs 0.7 $\mathrm{mg} / \mathrm{dL}$ [IQR 0.5-1.1], $\mathrm{p}<0.001)$. In the high-tier programs, recipients were more commonly blood type $\mathrm{O}$ (37.8\% vs $37.8 \%$ vs $41.2 \%, \mathrm{p}<0.001)$, have allograft failure requiring retransplantation $(2.8 \%$ vs $3.1 \%$ vs $3.8 \%, \mathrm{p}=0.017$ ), and to be status $1 \mathrm{~A}$ at the time of OHT (28.7\% vs $26.4 \%$ vs $29.7 \%, \mathrm{p}<0.001)$.

\section{Donor Characteristics Stratified by Center Acceptance Tercile}

Of donor hearts accepted and transplanted each tercile, high-tier centers more commonly utilized hearts from female donors (28.6\% vs $29.9 \%$ vs $31.1 \%, \mathrm{p}=0.03)$ (Supplemental Table 4 ). High-tier centers were also more likely to accept higher-risk donors including HCV, HBV, and HIV positive donors (2.8\% vs $3.2 \%$ vs $5.0 \%, \mathrm{p}<0.001)$, donors $>500$ miles from the transplant center $(2.6 \%$ vs $3.7 \%$ vs $6.5 \%, \mathrm{p}<0.001)$, and donors older than 40 years $(22.1 \%$ vs $23.3 \%$ vs $25.8 \%, \mathrm{p}<0.001)$. The rates of acceptance for donor hearts with LVEF $<60 \%$ was similar across the terciles $(\mathrm{p}=0.28)$.

\section{Multivariable Cox Regression for Mortality}

Cox Frailty modeling was conducted to investigate associations between center acceptance rates and ECD risk factors on overall mortality. In a multivariable model, higher tier centers with greater donor acceptance rates were not found to have significant impacts on mortality (mid-tier HR 0.91; 95\% CI 0.8-1.1; $\mathrm{p}=0.30$ and high-tier HR 0.9; 95\% CI 0.79-1.1; p=0.24) (Supplemental Table 5 ). With the exception of donor age $>40$ years, all ECD risk factors were not associated with hazards for mortality in univariate analysis (all, p>0.05), and were not included in the multivariable model. Recipients of donor hearts older than 
40 years had a $33 \%$ (HR 1.33, 95\% CI 1.22-1.46, p <0.001) increased hazard for posttransplant mortality. Other risk factors for posttransplant mortality included recipient creatinine $>1.5 \mathrm{mg} / \mathrm{dL}$ (HR 1.23; $95 \%$ CI 1.1-1.4; $\mathrm{p}<0.001$ ), graft ischemic time $>180$ minutes (HR 1.2; 95\% CI 1.1, 1.3; $\mathrm{p}=0.001$ ), and pre-OHT extracorporeal membrane oxygenator support (HR 2.3; 95\% CI 1.6, 3.1, p<0.001).

In a univariate analysis, donor acceptance tier was not associated with mortality across most ECD risk factors (over 50 offers, over 500 miles from the transplant center, and HCV, HBV or HIV positive) (all, p $>0.05$ ) (Supplemental Table 6 ). In donors with LVEF $<60 \%$, high-tier was associated with decreased hazards for mortality (HR 0.79; 95\% CI 0.63-0.00, $\mathrm{p}=0.039$ ).

\section{Estimated Longitudinal Survival}

Long-term Kaplan Meier survival rates among low-, mid-, and high-acceptance terciles were comparable at 1-year and 5-years (Figure 1a).

Of OHT recipients of whom accepted donors were $>40$ years old, 1 -year $(89.4 \%$ vs $88.3 \%$ vs $88.7 \%)$ and 5 year $(76.3 \%$ vs $74.8 \%$ vs $75.9 \%)$ posttransplant survival were comparable across acceptance terciles ( $\mathrm{p}=0.89)$ (Figure 1b ). For recipients of donors with LVEF <60\%, survival at 1-year (99.8\% vs $90.9 \%$ vs $91.6 \%$ ) and 5 -years $(78.4 \%$ vs $80.1 \%$ vs $80.9 \%)$ was similar across terciles ( $\mathrm{p}=0.12)$ (Figure 2a ). Recipients who were transplanted with donors from $>500$ miles from their transplant center also had comparable survival at 1 -year (92.7\% vs $91.6 \%$ vs $89.3 \%)$ and 5 -years $(78.5 \%$ vs $77.8 \%$ vs $77.7 \%)$ regardless of center volume tercile ( $\mathrm{p}=0.89$ ) (Figure 2b ). Furthermore, posttransplant survival from donors with $>50$ offers were also similar at 1 -year (92.6\% vs $95.2 \%$ vs $89.5 \%)$ and 5 -years ( $77.3 \%$ vs $80.3 \%$ vs $76.9 \%)$ among the three terciles $(\mathrm{p}=0.34)$ (Figure 3a ). Finally, recipients who were transplanted with HBV, HCV or HIV positive donor hearts also had comparable survival between the acceptance-based center terciles at 1-year $(85.0 \%$ vs $91.6 \%$ vs $91.0 \%)$ and 5 -years $(69.7 \%$ vs $72.4 \%$ vs $78.2 \%)$ post-OHT $(\mathrm{p}=0.24)$ (Figure $3 \mathbf{b}$ ).

\section{Discussion}

Waitlisted candidates often face certain disparities when seeking a donor heart for transplantation, both in organ availability and posttransplant outcomes. The etiology of these disparities is multifactorial, including blood type ${ }^{9}$, race and socioeconomic factors ${ }^{10}$, and even geographic features ${ }^{11}$. These disparities are likely driven and compounded by the relative scarcity of available organs for transplantation, as 3,500 OHTs were performed in the United States in $2019^{12}$ but an additional 3,500 remain waitlisted ${ }^{13}$. Furthermore, it is reasonable to suspect that a candidate's access to donor organs is also affected by their transplanting center's aggressiveness and likelihood to accept both traditional donors and ECDs. The transplanting provider team may face an interesting compromise during this selection process, balancing the pressure of selecting the "best" possible donor for each recipient, but also ensuring as many candidates are given the opportunity to receive a transplant.

The shortage of available donor organs has led to an increase in transplantation of donor organs outside of the standard criteria for organ donation (i.e. ECDs) with acceptable outcomes noted in the lung, kidney and liver transplant populations ${ }^{14-16}$. However, despite progress in other areas of organ transplantation, the outcomes following the utilization of ECDs in OHT have not been well described ${ }^{17}$. There is wide variability in organ acceptance practices at OHT centers across the United States and this has been shown to impact waitlist survival with candidates listed at centers with lower acceptance rates having higher waitlist mortality ${ }^{18}$. This variability demonstrates that the acceptability of certain organs is not well established, and while one center may accept the organ for OHT, another may reject, further reducing the potential donor pool. In fact, over $50 \%$ of donor hearts offered in the United States are rejected for $\mathrm{OHT}^{19}$. Donor hearts with unclear acceptability patterns tend to be outside of the traditionally accepted donor criteria but may still be potentially suitable for transplantation ${ }^{3}$. Furthermore, considering that centers with more aggressive donor acceptance rates have been shown to have superior post-OHT outcomes when compared to centers with lower volumes ${ }^{20}$, uncertainty exists as to if there is a volume-relationship between the use of ECDs in OHT and if an association exists between ECD recipient's outcomes. This study aims to address these remaining questions. 
This analysis highlights several interesting findings. First and less surprising, aggressive transplanting centers are more frequently utilizing donors with extended-criteria features. However, long-term results for these centers does not seem to be negatively impacted in comparison to centers with less frequent ECD usage. Second, in candidates who receive donation from a donor with extended-criteria features, long-term outcomes do not appear to be influenced by the aggressiveness and relative volume of the transplanting center. Lastly, in comparison to recipients of traditional criteria organs, recipients of ECD organs did not demonstrate any differences in overall survival in four of the five extended-criteria factors. Only donor age $>40$ years was found to have increased hazards for posttransplant mortality.

\section{Study Implications}

This study demonstrates that there is wide variability in the acceptance of extended criteria donors among low-, mid-, and high-tier centers, yet the survival rates of recipients of ECD hearts are comparable regardless of center volume/aggressiveness. These findings suggest that widespread adoption of higher ECD utilization practices may increase donor heart availability in the United States without an adverse impact on posttransplant outcomes. When evaluating donors with extended-criteria features, donor age appears to be the only factor with potential impact on long-term outcomes.

The donor selection process is admittedly variable and subjective to a multitude of factors. Centers often reflect on their recent outcomes and adjust aggressiveness in donor acceptance accordingly. For example, a center with several recent posttransplant deaths may become more conservative in selecting donors. This sometimes can make associations difficult to ascertain between center aggressiveness, transplant volume, and outcomes, as it can become unclear which factor contributed to the other. Nonetheless, there is a general understanding amongst physicians involved in OHT that considerable variability exists not only between programs but amongst providers in the same institution. Donor characteristics that may lead one center or provider to decline an offer are considered negligible by others. Further complicating this issue is the status of the recipient. Recipients who are declining clinically may have less time to wait for a suitable donor, thus making the center more aggressive in their donor acceptance behavior. The current analysis cannot account for all of these potential influencing factors but does ultimately suggest that centers who have higher donor acceptance rates and greater ECD utilization do not seem to be adversely impacted with regards to posttransplant outcomes of their recipients. A better in-depth understanding of donor selection criteria and practice by these centers with potential education of less aggressive programs may yield higher rates of OHT nationally without a detrimental impact on outcomes.

\section{Limitations}

This study was prone to limitations. First, this was a retrospective and non-randomized review of donor heart offers and acceptances. As such, its study design may be subject to selection bias. It is possible that some transplanting centers may receive disproportionately higher or lower quality heart offers, which may affect center acceptance rates and outcomes of this study. While there is no quantitative metric of overall quality of a heart offer, we did attempt to eliminate donor offers that were likely of poor quality as evidenced by receiving no acceptances of any solid organs from that donor. Furthermore, on multivariable analysis, we adjusted for possible confounding donor and recipient risk factors that may influence posttransplant survival. It is possible that additional unmeasured variables may have influenced these results. Lastly, to prevent possible bias from including highly inexperienced centers, we removed all centers that performed less than 10 transplants within the 5-year study period.

\section{Conclusions}

In this study of 85,505 donor heart offers across the United States to 133 different centers, 15,264 (17.9\%) resulted in acceptance for OHT. Centers range considerably with regards to donor heart acceptance rates. In centers with higher acceptance rates, there was a higher utilization of ECDs, yet one- and five-year posttransplant survival were comparable to less aggressive transplanting centers. Additionally, of the five ECD factors, only donor age $>40$ years was associated with higher risk for posttransplant mortality. This data suggests that transplanting centers, regardless of aggressiveness and clinical volume, can safely utilize 
ECDs with the other four risk factors (LVEF $<60 \%,>500$-mile distance, $>50$ previous offers, and HIV, HCV, or HBV positive) and achieve comparable posttransplant outcomes. Expansion of these donor criteria may help alleviate the relative organ shortage in the wake of an increasing heart failure population. Furthermore, a more in-depth understanding of donor acceptance practices in more aggressive centers with possible education and transfer of these practices to less aggressive centers may yield more OHTs nationally without an adverse impact on outcomes.

\section{Acknowledgements}

None

\section{Sources of Funding}

None

\section{Disclosures}

IS receives institutional research support from Medtronic and Atricure. AK is on the medical advisory board for Medtronic, Inc. These affiliations do not create direct conflicts with the content of this manuscript.

\section{References}

1. Khush KK, Cherikh WS, Chambers DC, et al. The International Thoracic Organ Transplant Registry of the International Society for Heart and Lung Transplantation: Thirty-sixth adult heart transplantation report - 2019; focus theme: Donor and recipient size match. J Hear Lung Transplant . 2019;38(10):10561066. doi:10.1016/j.healun.2019.08.004

2. Beuth J, Falter F, Pinto Ribeiro RV, Badiwala M, Meineri M. New strategies to expand and optimize heart donor pool: Ex vivo heart perfusion and donation after circulatory death: A review of current research and future trends. Anesth Analg . 2019;128(3):406-413. doi:10.1213/ANE.0000000000003919

3. Mori M, Wilson L, Ali A, et al. Evaluation of Case Volumes of a Heart Transplant Program and Shortterm Outcomes After Changes in the United Network for Organ Sharing Donor Heart Allocation System. JAMA Netw open . 2020;3(9):e2017513. doi:10.1001/jamanetworkopen.2020.17513

4. Kobashigawa J, Khush K, Colvin M, et al. Report From the American Society of Transplantation Conference on Donor Heart Selection in Adult Cardiac Transplantation in the United States. In: American Journal of Transplantation. Vol 17. Blackwell Publishing Ltd; 2017:2559-2566. doi:10.1111/ajt.14354

5. Menachem JN, Lindenfeld J, Schlendorf K, et al. Center volume and post-transplant survival among adults with congenital heart disease.J Hear Lung Transplant . 2018;37(11):1351-1360. doi:10.1016/j.healun.2018.07.007

6. Kilic A, Weiss ES, Allen JG, et al. Should orthotopic heart transplantation using marginal donors be limited to higher volume centers? Ann Thorac Surg . 2012;94(3):695-702. doi:10.1016/j.athoracsur.2012.03.069

7. Russo MJ, Iribarne A, Easterwood R, et al. Post-heart transplant survival is inferior at low-volume centers across all risk strata.Circulation . 2010;122(11 SUPPL. 1). doi:10.1161/CIRCULATIONAHA.109.926659

8. Iyengar A, Han J, Helmers M, et al. Relationship Between Change in Heart Transplant Volume and Outcomes: A National Analysis. J Card Fail . 2020;26(6):515-521. doi:10.1016/j.cardfail.2019.11.023

9. Ando M, Takeda K, Kurlansky PA, et al. Association between recipient blood type and heart transplantation outcomes in the United States.J Hear Lung Transplant . 2020;39(4):363-370. doi:10.1016/j.healun.2019.12.006

10. Singh TP, Naftel DC, Addonizio L, et al. Association of race and socioeconomic position with outcomes in pediatric heart transplant recipients. Am J Transplant . 2010;10(9):2116-2123. doi:10.1111/j.16006143.2010.03241.x 
11. Schulze PC, Kitada S, Clerkin K, Jin Z, Mancini DM. Regional differences in recipient waitlist timeand pre- and post-transplant mortality after the 2006 united network for organ sharing policy changes in the donor heart allocation algorithm. JACC Hear Fail . 2014;2(2):166-177. doi:10.1016/j.jchf.2013.11.005

12. Organ transplant trends | More transplants than ever | UNOS. https://unos.org/data/transplant-trends/. Accessed October 22, 2020.

13. Data - OPTN. https://optn.transplant.hrsa.gov/data/. Accessed October 22, 2020.

14. Christie IG, Chan EG, Ryan JP, et al. National Trends in Extended Criteria Donor Utilization and Outcomes for Lung Transplantation. Ann Thorac Surg . July 2020. doi:10.1016/j.athoracsur.2020.05.087

15. Pagano D, Barbara M, Seidita A, et al. Impact of Extended-Criteria Donor Liver Grafts on Benchmark Metrics of Clinical Outcome After Liver Transplantation: A Single Center Experience. Transplant Proc . 2020;52(5):1588-1592. doi:10.1016/j.transproceed.2020.02.050

16. Bonsignore P, Pagano D, Piazza S, et al. Crucial Role of Extended Criteria Donors in Deceased Donor Single Kidney Transplantation to Face Chronic Shortage in the Heart of the Mediterranean Basin: A SingleCenter Experience. Transplant Proc . 2019;51(9):2868-2872. doi:10.1016/j.transproceed.2019.02.075

17. Hsich EM. Matching the market for heart transplantation. Circ Hear Fail . 2016;9(4). doi:10.1161/CIRCHEARTFAILURE.115.002679

18. Choi AY, Mulvihill MS, Lee HJ, et al. Transplant Center Variability in Organ Offer Acceptance and Mortality among US Patients on the Heart Transplant Waitlist. JAMA Cardiol . 2020;5(6):660-668. doi:10.1001/jamacardio.2020.0659

19. Baran DA, Copeland H, Copeland J. What Number Are We?: Donor Sequence and Outcomes of Heart Transplantation. Circ Hear Fail . 2019;12(5). doi:10.1161/CIRCHEARTFAILURE.118.005823

20. Khush KK, Menza R, Nguyen J, Zaroff JG, Goldstein BA. Donor predictors of allograft use and recipient outcomes after heart transplantation. Circ Hear Fail . 2013;6(2):300-309. doi:10.1161/CIRCHEARTFAILURE.112.000165

Table 1. Total number of cardiac donor offers, acceptances, and refusals by year.

\begin{tabular}{|c|c|c|c|c|c|c|c|}
\hline & 2013 & 2014 & 2015 & 2016 & 2017 & 2018 & Total \\
\hline $\begin{array}{l}\text { Number } \\
\text { of trans- } \\
\text { planting } \\
\text { centers }\end{array}$ & 116 & 123 & 118 & 120 & 118 & 119 & 133 \\
\hline $\begin{array}{l}\text { Total } \\
\text { offers } \\
\text { among } \\
\text { centers }\end{array}$ & 14,327 & 15,821 & 12,738 & 13,634 & 14,422 & 14,563 & 85,505 \\
\hline $\begin{array}{l}\text { Total } \\
\text { offers } \\
\text { accepted } \\
\text { among } \\
\text { centers }\end{array}$ & 2,142 & 2,258 & 2,351 & 2,755 & 2,820 & 2,938 & 15,264 \\
\hline $\begin{array}{l}\text { Average } \\
\text { center }\end{array}$ & $\begin{array}{l}16.65 \% \\
(11.66)\end{array}$ & $\begin{array}{l}16.38 \% \\
(14.06)\end{array}$ & $\begin{array}{l}20.05 \% \\
(13.89)\end{array}$ & $\begin{array}{l}21.65 \% \\
(14.84)\end{array}$ & $\begin{array}{l}23.23 \% \\
(19.36)\end{array}$ & $\begin{array}{l}22.2 \% \\
(13.14)\end{array}$ & $\begin{array}{l}19.05 \% \\
(10.83)\end{array}$ \\
\hline
\end{tabular}




\begin{tabular}{|c|c|c|c|c|c|c|c|}
\hline & 2013 & 2014 & 2015 & 2016 & 2017 & 2018 & Total \\
\hline $\begin{array}{l}\text { Median } \\
\text { center } \\
\text { acceptance } \\
\text { rate, \% } \\
\text { (IQR) }\end{array}$ & $\begin{array}{l}13.65 \% \\
(9.40-21.21)\end{array}$ & $\begin{array}{l}12.80 \% \\
(8.69-21.72)\end{array}$ & $\begin{array}{l}17.62 \% \\
(11.42- \\
26.32)\end{array}$ & $\begin{array}{l}19.37 \% \\
(12.61- \\
28.81)\end{array}$ & $\begin{array}{l}19.21 \% \\
(12.07- \\
26.66)\end{array}$ & $\begin{array}{l}19.56 \% \\
(14.28- \\
26.87)\end{array}$ & $\begin{array}{l}16.85 \% \\
(12.06- \\
23.08)\end{array}$ \\
\hline $\begin{array}{l}\text { Number of } \\
\text { offers } \\
\text { refused }\end{array}$ & 10,746 & 11,636 & 8,987 & 9,408 & 10,066 & 9,860 & 60,703 \\
\hline $\begin{array}{l}\text { Offers } \\
\text { Refused } \\
\text { because of } \\
\text { donor age }\end{array}$ & $\begin{array}{l}6,064 \\
(56.43 \%)\end{array}$ & $\begin{array}{l}6437 \\
(55.32 \%)\end{array}$ & $\begin{array}{l}4760 \\
(52.96 \%)\end{array}$ & $\begin{array}{l}4936 \\
52.46 \%)\end{array}$ & $\begin{array}{l}5413 \\
(53.77 \%)\end{array}$ & $\begin{array}{l}5121 \\
(51.93 \%)\end{array}$ & $\begin{array}{l}32731 \\
(53.92 \%)\end{array}$ \\
\hline
\end{tabular}

IQR, interquartile range; SD, standard deviation

Table 2. Donor offers accepted by different donor characteristics

\begin{tabular}{lll}
\hline & Total Offers & Accepted Offers \\
\hline Age over 40 years & 39,955 & $3,697(9.25 \%)$ \\
LVEF <60\% & 28,798 & $4,445(15.44 \%)$ \\
Distance over 500 miles & 18,959 & $737(3.89 \%)$ \\
HIV, HBC, or HCV positive & 4,124 & $604(14.65 \%) ? ¿$ ? \\
50 total offers & 31,851 & $498(1.56 \%)$ \\
\hline
\end{tabular}

HBV, Hepatitis B virus; HCV, Hepatitis C virus; HIV, human immunodeficiency virus; LVEF, left ventricular ejection fraction

Table 3. Rates of extended-criteria donor acceptance by center tercile

\begin{tabular}{|c|c|c|}
\hline & Low-Tier & Mid-Tier \\
\hline Center Donor Acceptance, \% & $<13.71 \%$ & $13.71 \%-20.2$ \\
\hline Number of centers & 44 & 44 \\
\hline Total offers & 32,402 & 28,268 \\
\hline Donor age over 40 & Donor age over 40 & Donor age ove \\
\hline Total offers & $15,459(41.71 \%)$ & $13,613(48.16 \%)$ \\
\hline Acceptance frequency $(\%)$ & $752(4.86 \%)$ & $1,093(8.03 \%)$ \\
\hline Donor LVEF <60\% & Donor LVEF <60\% & Donor LVEF \\
\hline Total offers & $11,531(35.66 \%)$ & $9,264(32.77 \%)$ \\
\hline Acceptance frequency $(\%)$ & $1,026(8.88 \%)$ & $1,330(14.36 \%)$ \\
\hline Donor HIV, HBC, or HIV positive & Donor HIV, HBC, or HIV positive & Donor HIV, $\mathbf{F}$ \\
\hline Total offers & $1,384(4.27 \%)$ & $1,321(4.67 \%)$ \\
\hline Acceptance frequency $(\%)$ & $96(6.94 \%)$ & $151(11.43 \%)$ \\
\hline Donor with $>50$ offers placed & Donor with $>50$ offers placed & Donor with $>$ \\
\hline Total offers & $13,045(40.26 \%)$ & $10,993(38.89 \%)$ \\
\hline Acceptance frequency $(\%)$ & $81(0.62 \%)$ & $148(1.35 \%)$ \\
\hline Donor $>500$ miles from transplanting center & Donor $>500$ miles from transplanting center & Donor $>500 r$ \\
\hline Total offers & $6,738(20.80 \%)$ & $6,744(23.86 \%)$ \\
\hline Acceptance frequency (\%) & $90(1.34 \%)$ & $173(2.57 \%)$ \\
\hline
\end{tabular}


HBV, Hepatitis B virus; HCV, Hepatitis C virus; HIV, human immunodeficiency virus; LVEF, left ventricular ejection fraction

Table 4. Orthotopic heart transplant recipient outcomes stratified by center acceptance rate tercile

\begin{tabular}{lllll}
\hline & Low-Tier & Mid-Tier & High-Tier & P-Value \\
\hline Number of recipients & $\mathbf{3 , 4 1 4}$ & $\mathbf{4 , 6 8 3}$ & $\mathbf{7 , 1 2 5}$ & \\
Mortality & & & & \\
30-day & $122(3.57 \%)$ & $182(3.89 \%)$ & $245(3.44 \%)$ & 0.440 \\
1-year & $325(9.52 \%)$ & $406(8.67 \%)$ & $616(8.65 \%)$ & 0.293 \\
Long-term & $558(16.34 \%)$ & $692(14.78 \%)$ & $1053(14.78 \%)$ & 0.080 \\
Posttransplant follow up, years & $2.43(1.03-4.00)$ & $2.15(1.02-4.00)$ & $2.48(1.03-4.00)$ & \\
Re-transplant & $18(0.53 \%)$ & $20(0.43 \%)$ & $50(0.70 \%)$ & 0.142 \\
Median time to retransplant, years & $1.83(0.02-3.60)$ & $1.90(0.05-2.90)$ & $1.87(0.02-3.40)$ & \\
Hospital length of stay, days & $24.0 \pm 29.2$ & $22.3 \pm 24.3$ & $21.1 \pm 22.9$ & $<0.001$ \\
Average waitlist time, days & $337 \pm 442$ & $268 \pm 386$ & $188 \pm 325$ & $<0.001$ \\
Renal failure requiring dialysis & $429(12.57 \%)$ & $655(13.99 \%)$ & $882(12.38 \%)$ & 0.031 \\
Permanent pacemaker & $95(2.78 \%)$ & $142(3.03 \%)$ & $196(2.75 \%)$ & 0.647 \\
Stroke & $92(2.69 \%)$ & $144(3.07 \%)$ & $206(2.89 \%)$ & 0.601 \\
Reoperation & $126(3.69 \%)$ & $172(3.67 \%)$ & $255(3.58 \%)$ & 0.945 \\
One-year drug-treated acute rejection & $416(12.19 \%)$ & $601(12.83 \%)$ & $705(9.89 \%)$ & $<0.001$ \\
Drug-treated infection & $244(7.15 \%)$ & $335(7.15 \%)$ & $461(6.47 \%)$ & 0.252 \\
\hline
\end{tabular}

\section{Figure Legends:}

Figure 1. Kaplan Meier posttransplant survival comparison of donor acceptance terciles in a) overall all transplant recipients and b) recipients of donors greater than 40 years

Figure 2. Kaplan Meier posttransplant survival comparison of donor acceptance terciles in a) recipients of donors with left ventricular ejection fraction $<60 \%$ and b) recipients of donors greater than 500 miles from transplanting center

Figure 3. Kaplan Meier posttransplant survival comparison of donor acceptance terciles in a) recipients of donors who have received more than 50 offers and b) recipients of donors that are HIV, HBV, or HCV positive

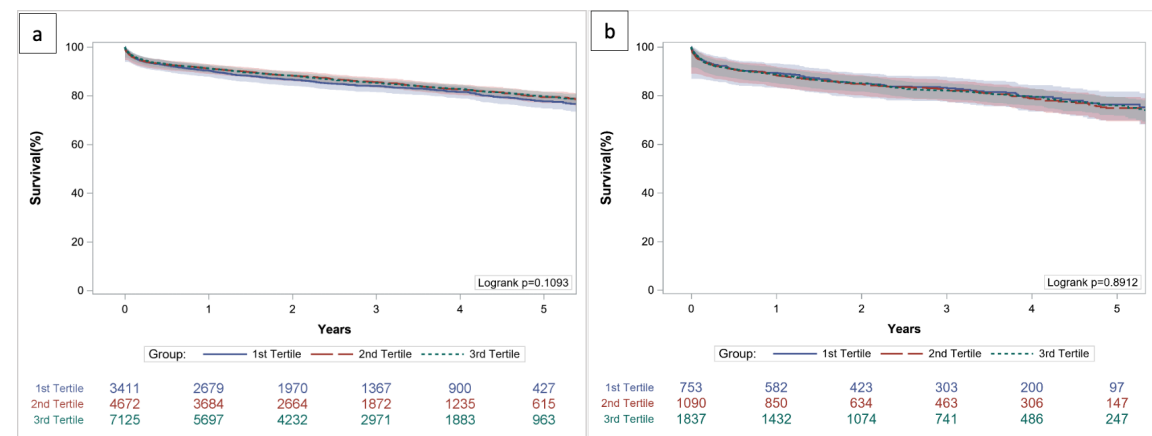



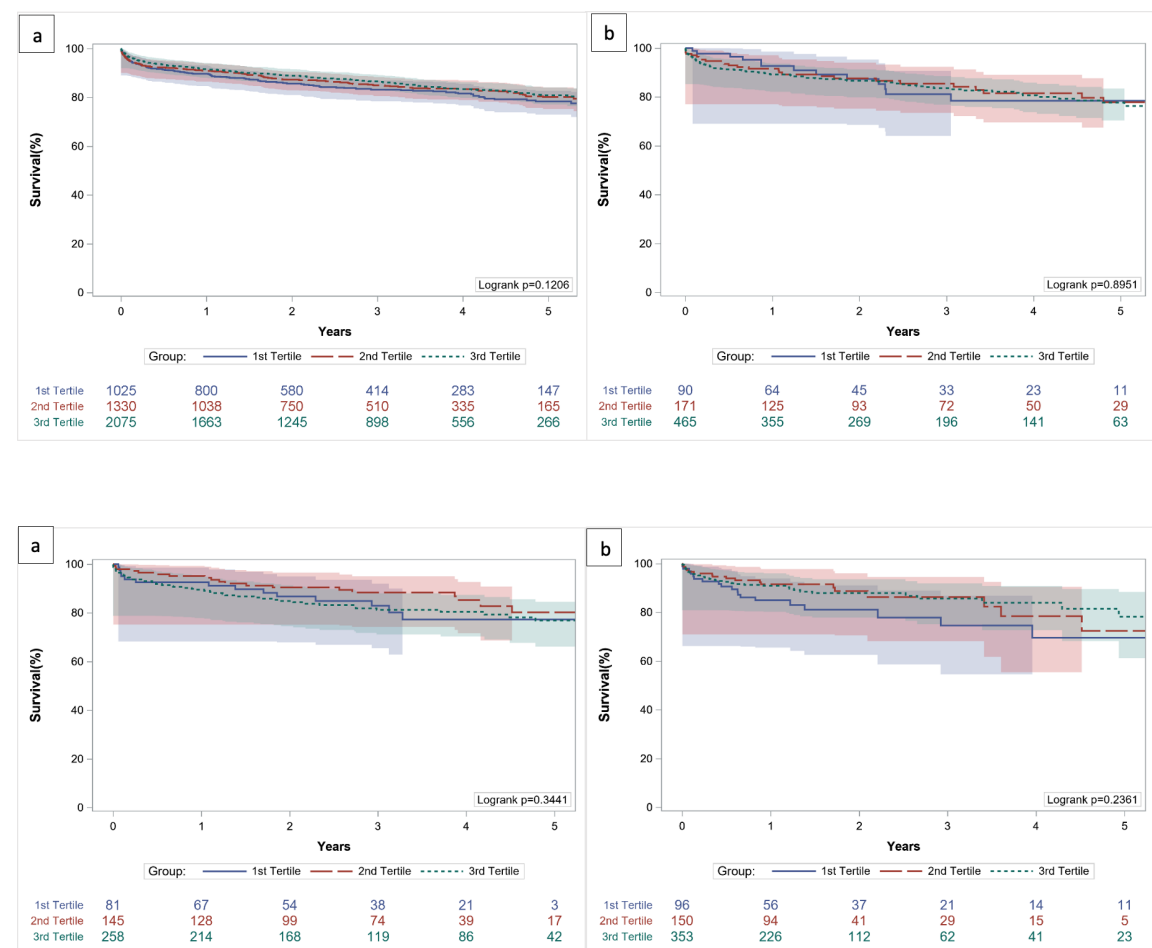